\title{
Phase Behavior and Rheology of SWNTs in Superacids
}

\author{
Virginia A. Davis, ${ }^{\dagger, \neq}$ Lars M. Ericson, ${ }^{\dagger, \S}$ A. Nicholas G. Parra-Vasquez, ${ }^{\dagger, \pm}$ Hua Fan, ${ }^{\dagger, \S}$ \\ Yuhuang Wang, ${ }^{\dagger, \perp}$ Valentin Prieto, ${ }^{\dagger,+} \mathrm{J}$ ason A. Longoria, ${ }^{\dagger,+}$ Sivarajan Ramesh,,,$\perp$ \\ Rajesh K. Saini, ${ }^{\dagger, \perp}$ Carter Kittrell, ${ }^{\dagger}$ W. E. Billups,,$\perp$, W. Wade Adams, ${ }^{\dagger}$ \\ Robert H. Hauge, ${ }^{\dagger, \perp}$ Richard E. Smalley, ${ }^{t, \xi, \perp}$ and Matteo Pasquali ${ }^{*, t, \neq}$
}

Carbon Nanotechnol ogy Laboratory, Center for Nanoscale Science \& Technology, and

Department of Chemical Engineering, MS-362, Department of Physics, MS-61, and

Department of Chemistry, MS-60, Rice University, 6100 Main St., Houston, Texas 77005

Received August 19, 2003; Revised Manuscript Received October 11, 2003

\begin{abstract}
Single-walled carbon nanotubes (SWNTS) can be dispersed at high concentration in superacids; the protonation of SWNTs sidewalls eliminates wall-wall van der Waals interactions and promotes the dispersion process. At very low concentration, SWNTs in superacids dissolve as individual tubes which behave as Brownian rods. At higher concentration, SWNTs form a highly unusual nematic phase consisting of spaghetti-like self-assembled supermolecular strands of mobile, solvated tubes in equilibrium with a dilute isotropic phase. At even higher concentration, the spaghetti strands self-assemble into a polydomain nematic liquid crystal. Upon the introduction of small amounts of water, the liquid crystal phase separates into needle-shaped strands ( $20 \mu \mathrm{m}$ long) of highly aligned SWNTs, termed alewives. Under anhydrous condition, the liquid crystalline phase can be processed into highly aligned fibers of pure SWNT without the aid of any surfactants or polymers.
\end{abstract}

\section{Introduction}

Theoretical calculations and experimental measurements on individual SWNTs and SWNT ropes indicate that macroscopic fibers and sheets of neat SWNTs will display remarkable electrical, thermal, and mechanical properties. $^{1-6}$ Neat, well-aligned SWNT fibers and sheets could be the ultimate building blocks of ultrastrong and ultralight materials for aerospace and aeronautical applications and could yield electromechanical actuators and sensors with unprecedented performance. ${ }^{1}$ M oving from self-assembled mi croscopic ropes (diameter $\sim 10$ to $100 \mathrm{~nm}$, length $\sim$ tens of micrometers) to continuous macroscopic fibers (diameter $\geq 10 \mu \mathrm{m}$, continuous length) requires the ability to make and process highly concentrated solutions or dispersions of pristine SWNTs. Since the scalable production of large quantities of SWNTs has been achieved, ${ }^{7-9}$ the lack of a liquid able to disperse significant amounts of pristine SWNTs has been the single most important roadblock to manufacturing macroscopic articles composed solely of SWNTs. SWNTs have been dispersed at moderate concentrations $\sim 1$ vol \% with the aid of surfactants; ${ }^{10}$ however, this route requires large amounts of surfactant, some of which remains in the final product. ${ }^{11-13}$ Removal of the surfactant is not likely to be feasible in large-scale manufacturing processes.

We have successfully dispersed SWNTs at concentrati ons up to 10 wt \% in superacids, e.g., sulfuric acid with various levels of excess $\mathrm{SO}_{3}$, chlorosulfonic acid, and triflic acid. This concentration is over 10 times the highest concentration ever achieved with wrapping or stabilization by surfactants. ${ }^{1,10-12}$ The high concentrations achievable in superacids are due to the protonation of the SWNTs by a mechanism similar to that occurring

† Carbon Nanotechnology Laboratory, Center for Nanoscale Science \& Technology.

₹ Department of Chemical Engineering.

$\S$ Department of Physics.

$\perp$ Department of Chemistry.

* Corresponding author: e-mail mp@rice.edu. in rodlike polymers. ${ }^{14}$ The tubes are stabilized against aggregation by the formation of an electrostatic double layer of protons and negative counterions $\left(\mathrm{HSO}_{4}{ }^{-}\right.$in sulfuric acid). ${ }^{15}$

\section{Experimental Methods}

The SWNTs used in this study were produced from the highpressure carbon monoxide (HiPco) process at Rice University ${ }^{7,8}$ and purified according to literature methods. ${ }^{16}$ Oleum (20 wt $\%$ excess $\mathrm{SO}_{3}$, density $1925 \mathrm{~kg} / \mathrm{m}^{3}$ ), chlorosulfonic acid, and triflic acid were used as received from Sigma Aldrich. 102\% sulfuric acid ( 2 wt \% excess $\mathrm{SO}_{3}$ ) was prepared by mixing the ol eum with $96 \%$ sulfuric acid in the ratio $1.1 \mathrm{~mL}$ of oleum $/ \mathrm{mL}$ of $\mathrm{H}_{2} \mathrm{SO}_{4} .102 \%$ sulfuric acid was chosen to ensure that the solubility of the SWNTs would not be affected by the uptake of small amounts of moisture from the environment. ${ }^{15}$ Dispersions containing less than 0.5 wt \% SWNT were prepared by mixing with a magnetic stir bar for a minimum of 3 days in an anhydrous environment in a glovebox at room temperature. More concentrated dispersions were mixed in an argonblanketed double-helix mixer (Daca Instruments, Goleta, CA) at $110{ }^{\circ} \mathrm{C}$ for a minimum of 3 days. All glassware was flamedried before use, and the SWNTs were dried in the mixer under vacuum overnight at $80-100^{\circ} \mathrm{C}$ before acid was added. Volume fractions of SWNTs were calculated on the basis of a SWNT density of $1450 \mathrm{~kg} / \mathrm{m}^{3}$, which is typical of $\mathrm{HiPcO}$ SWNTs. The measured viscosity and density of the $102 \%$ sulfuric acid were respectively $26 \mathrm{mPa}$ s and $1880 \mathrm{~kg} / \mathrm{m}^{3}$ at $25^{\circ} \mathrm{C}$.

Rheol ogical measurements were made on ARES and RDA III strain-controlled rotational rheometers (Rheometrics Scientific, Piscataway, NJ, now TA Instruments, New Castle, $D E)$. Testing fixtures were made of Hastelloy $C$ or stainless steel 316 to avoid corrosion. Fixture geometries were a large Couette (i.d. $=32 \mathrm{~mm}$, o.d. $=34 \mathrm{~mm})$, parallel plates $(25 \mathrm{~mm}$ diameter), and cone and plate ( $25 \mathrm{~mm}$ diameter, cone angle = $0.04 \mathrm{rad}$ ). Generally, the Couette was used for testing concentrations below $0.5 \mathrm{wt} \%$, and cone and plate or parallel plates were used for testing concentrations above 0.5 wt \%. However, several concentrations were tested in multiple fixtures to ensure there were no artifacts in the data due to testing geometry. An anhydrous environment was maintained during loading and testing of all samples by continuous flow of argon into a custom-made environmental control chamber enclosing 


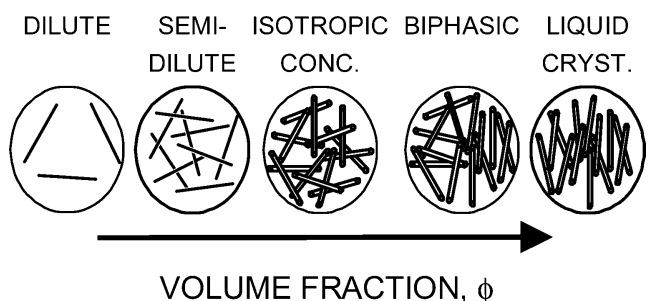

Figure 1. Phase behavior of solutions of Brownian rigid rods. $^{21,22}$

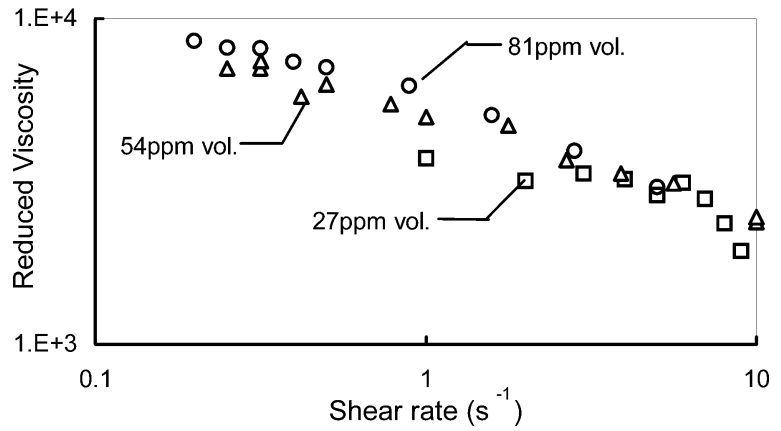

Figure 2. Reduced viscosity $[\eta]=\left(\eta-\eta_{\mathrm{s}}\right) /\left(\eta_{\mathrm{s}} \phi\right)$ vs shear rate in the dilute regime (from 27 to $81 \mathrm{ppm}$ vol). The data overlap well, in accordance with the theory of dilute Brownian rods. Because the polydispersity of the sample is unknown and it is estimated to be large, 10,24 it is not possible to determine the rotational relaxation time of the rods from these data.

the fixture. To provide additional protection against moisture ingress, the sample surface was covered with an inert lowviscosity fluid, Flourinert FC-77 (3M Corp., St. Paul, MN).

Optical microscopy was performed on a Zeiss Axioplan optical microscope using flame-dried glass slides and coverslips assembled in a glovebox. Scanning electron microscopy was performed a Phillips FEI XL30 eSEM without any coating.

\section{Results and Discussion}

Rheology and microscopy show that SWNTs in superacids roughly parallel the phase behavior of rodlike polymer solutions (such as poly(p-phenylene terephthalamide) (PPTA, i.e., Kevlar) in sulfuric acid or poly(benzobisoxazole) (PBO) in methanesulfonic acid) used for solution spinning of high-performance fibers from nematic liquid crystals. ${ }^{17-19}$ The distinguishing feature is that SWNTs in superacids self-assemble into extremely long strandlike structures and that this structure is the basic component of the liquid crystalline phase.

The phase behavior of rodlike polymer solutions is depicted in Figure 1. With increasing concentration, such systems transition from a dilute solution where individual rods do not interact with each other, to a semidilute solution where rotation is inhibited, to an isotropic concentrated solution where both translation and rotation are inhibited. At concentrations above the percolation threshold, steric effects force the system to phase separate into a liquid crystalline phase in equilibrium with the isotropic concentrated phase. ${ }^{20,21}$ Raising the concentration further increases the proportion of the liquid crystalline phase until the system becomes fully liquid crystalline.

Dilute Regime. Figure 2 shows a collapse of reduced viscosity $[\eta] \equiv\left(\eta-\eta_{\mathrm{s}}\right) /\left(\eta_{\mathrm{s}} \phi\right)$ vs shear rate $\dot{\gamma}$ for the three most dilute solutions $(27,54$, and $81 \mathrm{ppm}$ vol in $102 \%$ sulfuric acid). Measurements at 54 and $81 \mathrm{ppm}$ vol overlap al most perfectly; those at 27 ppm vol agree less well. Data at 27 ppm contain more error because the viscosity of the solution was only $\sim 10 \%$ higher than the solvent viscosity. This behavior of the reduced viscosity shows that the viscosity depends linearly on concentration and that the relaxation time is independent of concentration, in accordance with the theory of dilute Brownian rods; 23 thus, bel ow $\sim 90 \mathrm{ppm}$ vol the tubes are dissolved as individuals and do not interact.

The aspect ratio of a sample of Brownian rods can be extracted from the limiting value of the reduced viscosity at low shear rate. Figure 2 shows that shear thinning is incipient at $0.3 \mathrm{~s}^{-1}$, near the lowest shear rates that can be measured within the limit of the torque transducer. In the analysis below, the viscosity measured at the lowest accessi ble shear rate $\left(0.3 \mathrm{~s}^{-1}\right)$ is taken as the approximate value of the zero-shear viscosity.

The zero-shear viscosity of a (monodisperse) solution or dispersion of Brownian rigid rods is ${ }^{25}$

$$
\eta_{0} \equiv \lim _{\dot{\gamma} \rightarrow 0} \eta(\dot{\gamma})=\eta_{\mathrm{s}}\left(1+\frac{4}{5} \mathrm{k}_{\mathrm{B}} \mathrm{T} \tau v\right)
$$

where $\eta_{\mathrm{S}}$ is the solvent viscosity, $\mathrm{k}_{\mathrm{B}}$ is Boltzmann's constant, $\mathrm{T}$ is temperature, $v$ is the number of rods per unit volume

$$
\tau \equiv \frac{\varsigma^{3}}{72 \mathrm{k}_{\mathrm{B}} \mathrm{T}}
$$

is the rotational relaxation time of the rods, and $\varsigma$ is the perpendicular drag coefficient of the rods. The number concentration of rods is related to the volume fraction $\phi$ as $v \equiv \phi /\left(\pi \mathrm{R}^{2} \mathrm{~L}\right)$, where $\mathrm{R}$ is the rod radius and $L$ is the length. The perpendicular drag coefficient $\varsigma$ on a slender cylinder is ${ }^{26}$

$$
\varsigma=4 \pi \eta_{\mathrm{s}} \epsilon \mathrm{f}(\epsilon)
$$

where

$$
\begin{gathered}
\epsilon=\frac{1}{\ln (L / R)} \\
f(\epsilon)=\frac{1+0.64 \epsilon}{1-1.5 \epsilon}+1.659 \epsilon^{2}
\end{gathered}
$$

This yields the relationship

$$
\frac{\eta-\eta_{\mathrm{s}}}{\eta_{\mathrm{s}} \phi}=\frac{2(\mathrm{~L} / \mathrm{R})^{2}}{45[\ln (\mathrm{L} / \mathrm{R})]}\left[\left(\frac{1+\frac{0.64}{\ln (\mathrm{L} / \mathrm{R})}}{1-\frac{1.5}{\ln (\mathrm{L} / \mathrm{R})}}\right)+\frac{1.659}{(\ln (\mathrm{L} / \mathrm{R}))^{2}}\right]
$$

In the polydisperse case, the viscosity contributions of each subpopulation of length are additive, and the intrinsic viscosity is related to the ratio of the third and first moments of the distribution of length. The extrapolated value of the intrinsic viscosity is $\left[\eta_{0}\right] \equiv \lim _{\dot{\gamma} \rightarrow 0}(\eta(\dot{\gamma})$ $\left.-\eta_{\mathrm{s}}\right) /\left(\eta_{\mathrm{s}} \phi\right)=8300 \pm 830$ (Figure 2); taking this extrapolated value, eq 6 yields an average aspect ratio $\mathrm{L} / \mathrm{d}=470 \pm 30$. This result compares well with recent viscosity ${ }^{27}$ and $A F M^{10}$ measurements of individual tubes stabilized in pluronic surfactants. Another way of estimating the critical volume fraction for the transition from di lute to semi dilute is to take the reciprocal of the intrinsic viscosity; this estimate yiel ds a critical volume fraction of $120 \mathrm{ppm}$ vol, which compares well to the value of $90 \mathrm{ppm}$ obtained from the overlap shown in Figure 2. In terms of number of rods per unit sphere 


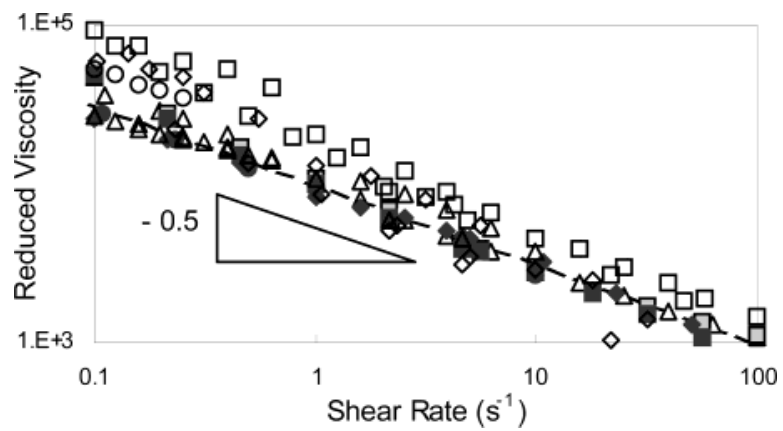

Figure 3. Reduced viscosity, $\left(\eta-\eta_{\mathrm{s}}\right) /\left(\eta_{\mathrm{s}} \phi\right)$, vs shear rate for 94-452 ppm vol. SWNTs in $100 \% \mathrm{H}_{2} \mathrm{SO}_{4}$ : filled symbols, $94-$ 454 ppm vol. SWNT batch HPR 105 September 2002; hollow symbols, February 2003 103-407 ppm vol. SWNT batch HPR 106.2. Slope of -0.5 is drawn for reference and agrees with the theory of K irkwood and Plock. Vertical offset between HPR 105 and HPR 106.2 may be partly due to small differences in the initial volumetric dilution.

swept by a rod, the transition concentrations are estimated to be $v \mathrm{~L}^{3} \equiv(4 / \pi) \phi(\mathrm{L} / \mathrm{d})^{2}=25-33$, which compares well with literature values. ${ }^{28}$

The specific intrinsic viscosity of the SWNTs is $\left[\eta_{0}\right]_{c}$ $=\left[\eta_{0}\right] / \rho_{\mathrm{b}} \approx 5.7 \mathrm{~m}^{3} / \mathrm{kg}=57 \mathrm{dL} / \mathrm{g}$; this value is comparable to or greater than the highest reported values for high molecular weight poly(p-benzamide) (PBA) in sulfuric $\operatorname{acid}^{29}\left(\left[\eta_{0}\right]_{c}=12.5 \mathrm{dL} / \mathrm{g}\right.$ for $\mathrm{M}_{\mathrm{w}}=64400 \mathrm{~g} / \mathrm{mol}$, i.e., $\mathrm{L} \approx$ $400 \mathrm{~nm}$ ), poly(benzobisthiazole) (PBT) in methanesulfonic acid ${ }^{30}\left(\left[\eta_{0}\right]_{\mathrm{c}}=26.5 \mathrm{dL} / \mathrm{g}\right.$ for $\mathrm{M}_{\mathrm{w}}=37000 \mathrm{~g} / \mathrm{mol}$, i.e., $\mathrm{L} \approx 190 \mathrm{~nm}$ ), large poly(benzobisoxazole) (PBO) in methanesulfonic acid ${ }^{31}\left(\left[\eta_{0}\right]_{\mathrm{c}}=50 \mathrm{dL} / \mathrm{g}\right.$ for $\mathrm{M}_{\mathrm{w}} \approx 230000$ $\mathrm{g} / \mathrm{mol}$, i.e., $\mathrm{L} \approx 390 \mathrm{~nm}$ ), and very large molecules of xanthan ${ }^{32}\left(\left[\eta_{0}\right]_{\mathrm{c}}=71 \mathrm{dL} / \mathrm{g}\right.$ for $\mathrm{M}_{\mathrm{w}} \approx 5.4 \mathrm{mg} / \mathrm{mol}$, i.e., $\mathrm{L}$ $\approx 2.78 \mu \mathrm{m})$.

Rods shear thin when the product of the longest rotational relaxation time $\tau$ and shear rate $\dot{\gamma}$ exceeds $\sim 0.2$ (i.e., the Weissenberg number $\mathrm{Wi} \equiv \tau \dot{\gamma}>0.2$ ); because shear thinning starts at $\dot{\gamma} \approx 0.3 \mathrm{~s}^{-1}$, we estimate that the longest rotational relaxation time is approximately $0.7 \mathrm{~s}$. The estimated value of the longest relaxation time together with eq 2 yields an estimated length $\mathrm{L} \approx 1.5 \mu \mathrm{m}$ for the longest nanotubes present in the $\mathrm{HiPco}$ sample (the diameter is nearly immaterial for the determination of the relaxation time).

Semidilute Regime. The measurements at concentrations between 94 and 600 ppm vol can be collapsed onto a separate master curve of reduced viscosity vs shear rate (Figure 3). Two separate HiPco batches are shown (HPR 105 and 106.2): the SWNTs produced in batch 106.2 display slightly higher reduced viscosity.
At low shear rates, these data depart from the dilute master curve (Figure 2 ) because the relaxation time is now concentration-dependent, and thus the viscosity starts shear thinning at lower shear rates (below the sensitivity of the rheometer transducer). In this concentration range, the solutions relax slower as concentration increases, as measured by the delay time between transient tests required to get reproducible viscosity data, indicating that $94-600 \mathrm{ppm}$ vol is in the semidilute regime.

Biphasic Regime. Optical microscopy shows that at $1000 \mathrm{ppm}$ vol (0.1 vol \%) the system is biphasic. The morphology of SWNTs in this bi phasic region is remarkable. The SWNTs self-assemble into seemingly endless strands of supermolecular aggregates. These strands are in equilibrium with the isotropic phase and are termed hereafter "SWNT spaghetti". The SWNT spaghetti are nematic liquid crystalline domains where tubes are free to translate along the length of the strand (Figure 4). Imaging successive z-planes shows that the strands continue through multiple focal planes and fails to reveal any ends. The constant optical density along the length of the strand suggests that the spaghetti have a uniform diameter bel ow $600 \mathrm{~nm}$ (part of the measured diameter is due to diffraction). At room temperature, the SWNT spaghetti phase first appears between 600 and $1000 \mathrm{ppm}$ vol. A more precise determination is difficult because at lower concentration it is necessary to scan at high magnification increasingly larger regions of the sample. The biphasic region is expected to be quite wide because the SWNTS are polydisperse in length and diameter. $10,28,33$

On the basis of the minimum of the viscosity vs concentration curve (Figure 6) and on measurements on solid structures induced by phase separation (Figure 10 and also ref 34), we believe that the SWNTs in the spaghetti phase are spaced by approximately $2-3 \mathrm{~nm}$, i.e., by roughly four to six molecules of sulfuric acid, and that they are highly aligned because of their stiffness and because of the steric constraints present on narrowly spaced object of such high aspect ratio. Presently, we cannot explain the peculiar shape of the SWNT spaghetti nematic phase; it may be related to the large polydispersity of the sample (the ratio between the length of the longest and shortest SWNTs is at least 20) and also to the differences between SWNTs and rodlike polymers. SWNTs have much higher bending stiffness than rodlike polymers (see Appendix A); they have cylindrical rather than locally planar cross section and in the absence of acid have a high van der Waals
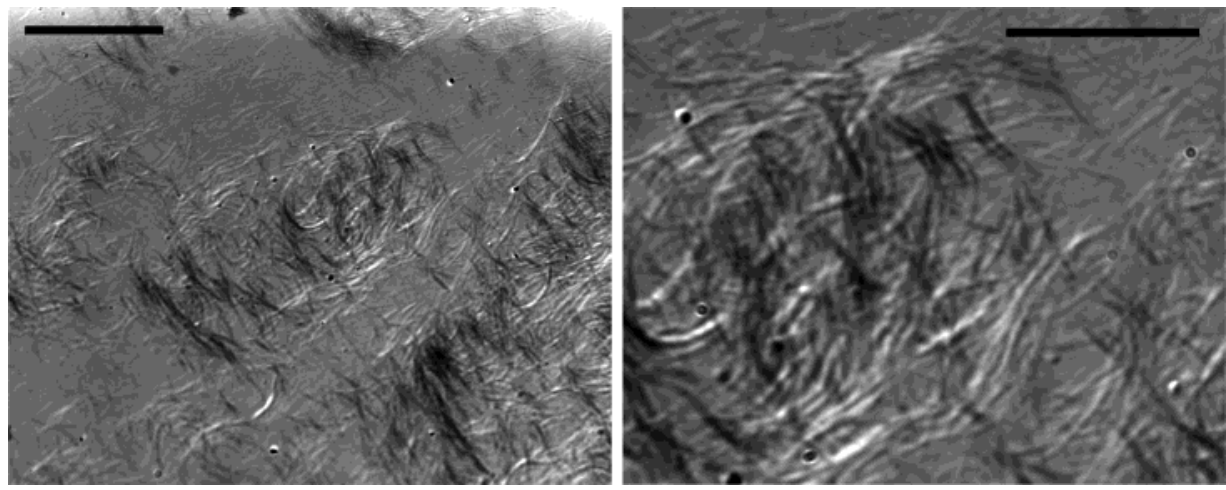

Figure 4. (left) Spaghetti in $0.32 \mathrm{vol} \%\left(0.25\right.$ wt \%) solution of SWNTs in $\mathrm{H}_{2} \mathrm{SO}_{4}$ imaged on a Zeiss Axioplan microscope with $63 X$ DIC, 1.4 NA oil immersion objective with $2.5 \times$ magnification in front of the camera. The scale bar is $20 \mu \mathrm{m}$. (right) A digitally enhanced, magnified section of the image at left, showing the size and uniformity of the spaghetti. The scale bar is $10 \mu \mathrm{m}$. 


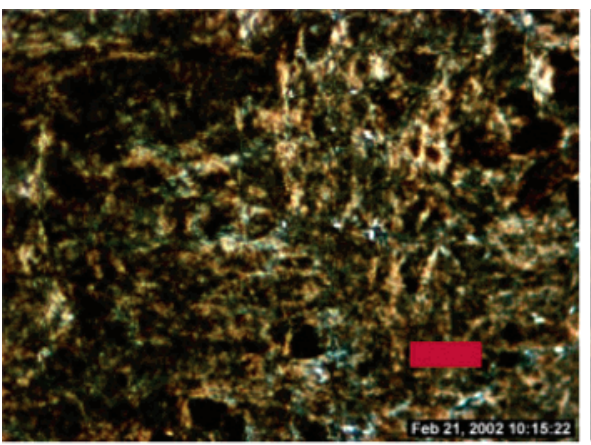

$0^{\circ}$

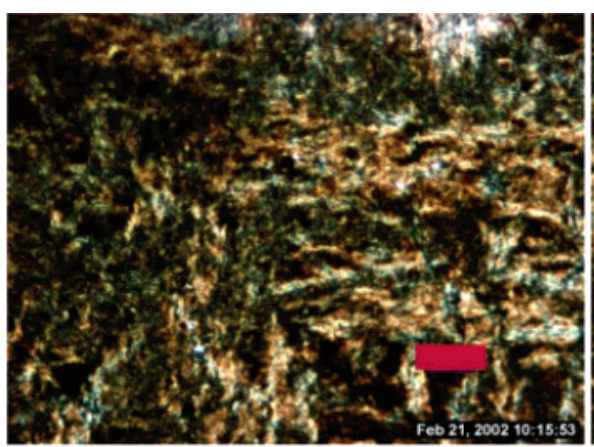

$45^{\circ}$

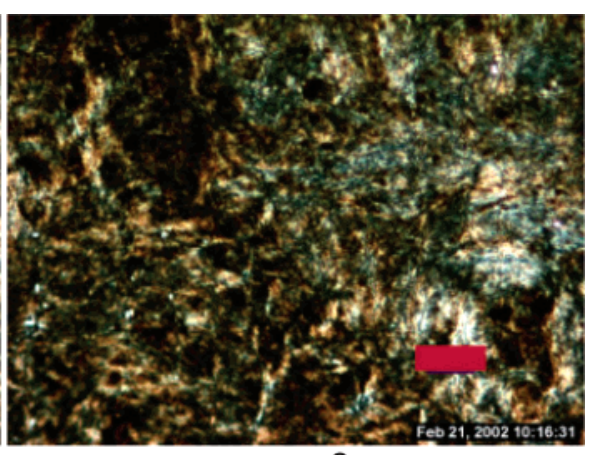

$90^{\circ}$

Figure 5. 5.1 vol \% (4 wt \%) SWNT under cross-polarized light on a Nikon E600 microscope with 20x NA 0.5 objective. The polydomains become bright and dim as the sample is rotated. The scale bar in the figures is $250 \mu \mathrm{m}$.

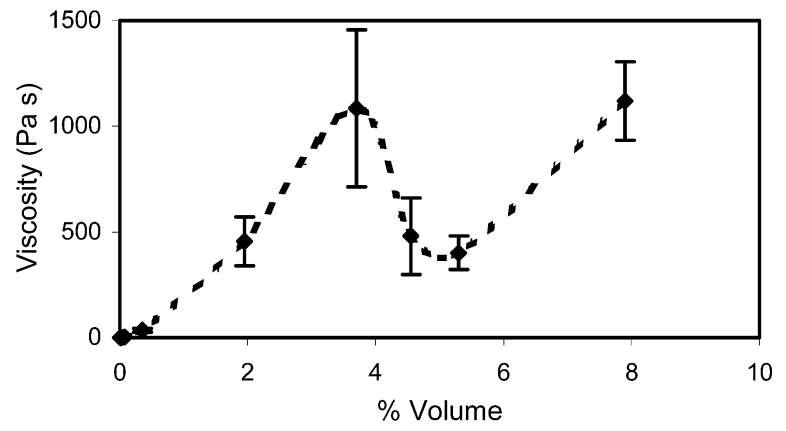

Figure 6. Relationship between viscosity and concentration for SWNTs in $102 \% \mathrm{H}_{2} \mathrm{SO}_{4}$ at a shear rate of $0.1 \mathrm{~s}^{-1}$. The system transitions to a single-phase liquid crystal between the maximum at $\sim 3.5 \mathrm{vol} \%$ and the minimum at $\sim 5.1 \mathrm{vol} \%$. The error bars represent the standard deviation obtained by measuring at least three different samples.

attraction of $\sim 0.5 \mathrm{eV} / \mathrm{nm}$ between side walls. The morphology of the spaghetti phase may be related to the molecular stiffness and geometry or to residual longrange attractive intermolecular forces in the acids.

Single-Phase Nematic Liquid Crystal: Optical Microscopy. Optical microscopy shows that the fraction of the SWNT spaghetti phase increases as the bulk concentration of SWNTs is raised, until only the SWNT spaghetti is present, yielding a single phase polydomain lyotropic nematic liquid crystal at approximately 4 vol $\%$ in $102 \%$ sulfuric acid. This single phase shows strong birefringence under polarized light (Figure 5). The polydomain structure is evidenced by rotating the sample between cross polars and observing that different domains turn bright and dark based on the relative alignment between their director and the polarization vector of the light. We believe that the polydomain structure results from the local coalescence of spaghetti strands that have ordered. On large length scales ( 100 $\mu \mathrm{m})$, the domains are randomly oriented with respect to each other. The healing time for defects at the boundaries of the spaghetti strands is expected to be quite long and can be estimated by using typical properties of nematic liquid crystalline polymers. The healing time $t_{a}$ for the spontaneous disappearance of a defect from a nematic liquid crystalline polymer of viscosity $\eta$ and thickness $h$ is

$$
\mathrm{t}_{\mathrm{a}} \approx \frac{\eta \mathrm{h}^{2}}{\mathrm{~K}}
$$

where $\mathrm{K}$ is the characteristic Frank constant. ${ }^{28}$ The viscosity of nematic SWNT/acid dispersions is between
400 and $500 \mathrm{~Pa}$ s. The effective "director diffusivity", $\mathrm{K} / \eta$, of SWNTs is unknown; typically it ranges between $\mathrm{K} / \eta \sim 10^{-8}$ and $10^{-10} \mathrm{~cm}^{2} / \mathrm{s}$ in polymeric nematics; 28 thus, a $500 \mu \mathrm{m}$ thick sample would take nearly 1 year to form a uniform monodomain liquid crystal.

Single-Phase Nematic Liquid Crystal: Rheology. The nematic nature of SWNT/superacid solutions is evidenced further by key rheological signatures unique to lyotropic liquid crystals formed by highmolecular-weight rodlike molecules: the viscosityconcentration curve has a maximum; the viscosity vs shear rate curve often shows three distinct regions, usually termed regions I, II, and III; with increasing shear rate, the first normal stress difference $\mathrm{N} 1$ changes sign from positive to negative and back to positive; in transient shearing tests the shear stress and first normal stress difference oscillate for $\sim 100$ shear units or more before reaching steady state; and the Cox-Merz rule is not obeyed.

Figure 6 shows that SWNTs in superacids exhibit this nonmonotonic relationship between viscosity and concentration. The transition from a biphasic system to a single-phase liquid crystal occurs in the concentration range between the maximum and the minimum in viscosity; 35 thus, the single-phase nematic liquid crystal forms between 3.5 and 5.1 vol \%, a slightly higher concentration than that estimated by Flory theory $(12.5 \mathrm{~d} / \mathrm{L} \sim 2.7 \mathrm{vol} \%$ based on an average aspect ratio of $L / d \approx 470$ ). Experimental observations on other systems have shown that Flory theory typically underpredicts the transition to a single-phase liquid crystal. ${ }^{33,35-41}$

Figure 7 shows the viscosity $\eta$ and first normal stress difference N1 vs shear rate curve of SWNT/superacid solutions at $4.5 \mathrm{vol} \%$, where the dispersion is predominantly or wholly anisotropic. Notably, the viscosity shear thins at low shear rate, then seems to plateau at $\dot{\gamma} \approx 1 \mathrm{~s}^{-1}$, and then shear thins again at high shear rates; the first normal stress difference is negative at shear rates above $1 \mathrm{~s}^{-1}$. The three-region shear viscosity curve was first observed by Onogi and Asada; 42 it is generally associated with tumbling nematics and is not exhibited by all Iyotropic nematic polymeric liquid crystals. ${ }^{40}$ The origin of the three region curve is still a subject of investigation and debate. One explanation is that the first shear thinning region, region $\mathrm{I}$, is a result of tumbling and the evolution of texture. At intermediate shear rates (region II) there is a competition between tumbling and the steady al ignment of the director with flow; as a result, the rods "wag" about a single director. At higher shear rates, region III, the director aligns in 


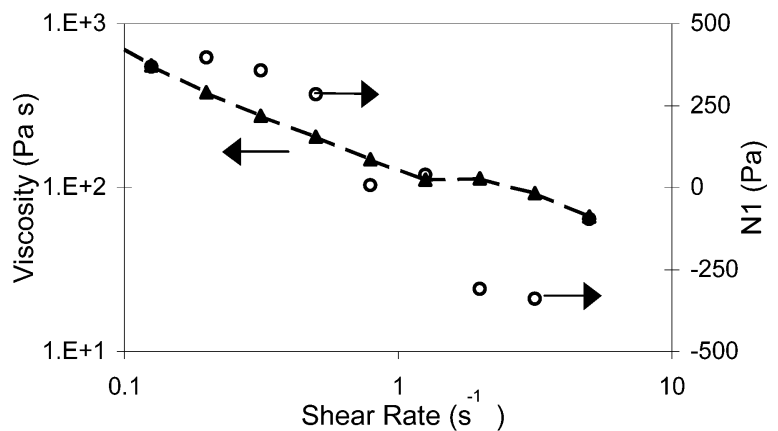

Figure 7. Viscosity vs shear rate for 4.5 vol \% (3.5 wt \%) SWNT in $102 \% \mathrm{H}_{2} \mathrm{SO}_{4}$ measured on $25 \mathrm{~mm}$ cone and plate (cone angle $=0.04 \mathrm{rad}$ ). N 1 is the thrust on the plate divided by the plate area. The behavior is indicative of the three-region behavior seen with some liquid crystalline polymers and shear alignment at shear rates $<10 \mathrm{~s}^{-1}$. The sign changes in first normal stress difference are also characteristic of liquid crystallinity.

the flow direction, resulting in shear thinning. $28,40,43$ SWNTs in $102 \% \mathrm{H}_{2} \mathrm{SO}_{4}$ display nearly constant viscosity in a narrow region of shear rates around $1 \mathrm{~s}^{-1}$, which indicates that there may be a very narrow region II plateau between the two shear thinning regions. Region III (shear-aligning behavior) begins at shear rate $\approx 3$ $\mathrm{s}^{-1}$, indicating that moderate shear should be sufficient to align nematic domains of SWNTs. The facile alignment is manifested by the high degrees of collective alignment in SWNT fibers produced by simply pushing the SWNT/superacid dispersion through a syringe needle into a coagulation bath, 34,44 as shown in Figure 10. As the shear rate exceeds a critical value (about $10 \mathrm{~s}^{-1}$ for $4.5 \mathrm{vol} \%$ ), the SWNT/superacid dispersion ejects itself from the rheometer gap.

The negative first normal stress difference N1 displayed by SWNT dispersions (Figure 7) is generally ${ }^{69}$ associated with lyotropic nematic liquid crystalline polymers. ${ }^{28}$ At low shear rate, N1 is positive and increases with shear rate as observed in polymer solutions. At a critical shear rate, N1 suddenly drops and switches to a large negative value. As shear rate is increased further, N1 turns positive again. 35,38,43,50 The origin of negative N 1 has been the subject of considerable research and is still not completely understood. ${ }^{28,38,51-53}$ Essentially, negative N1 is believed to be a consequence of director tumbling. For systems where negative N1 is observed, the change from positive to negative occurs in region II as a consequence of the transition from "tumbling" to "wagging". 54 In the shear aligning region, regi on III, N1 becomes positive again.

Figure 8 shows that the response of 7.6 vol \% SWNT/ superacid to start-up of steady shearing at shear rate 5 $\mathrm{s}^{-1}$. Both the shear stress and the first normal stress difference oscillate for hundreds of shear units (product of shear rate and time). This behavior is also typical of lyotropic liquid crystall ine polymers and quite different from that of isotropic suspensions and polymer solutions, where oscillatory transients last a few shear units at most. ${ }^{55}$ Long oscillatory transients were displayed by all concentrated samples ( $\phi>4.5 \mathrm{vol} \%$ ).

A final rheological signature demonstrated by SWNT/ acid sol utions is that the steady and dynamic viscosities do not exhibit the same behavior as a function of shear rate and frequency; ${ }^{50,55}$ i.e., the Cox-Merz rule is not obeyed (Figure 9). This empirical rule states that at a given frequency $\omega$ and shear rate $\dot{\gamma}$ the dynamic viscosity $\eta^{*}(\omega)$ and the steady shear viscosity $\eta(\dot{\gamma} Z)$ are

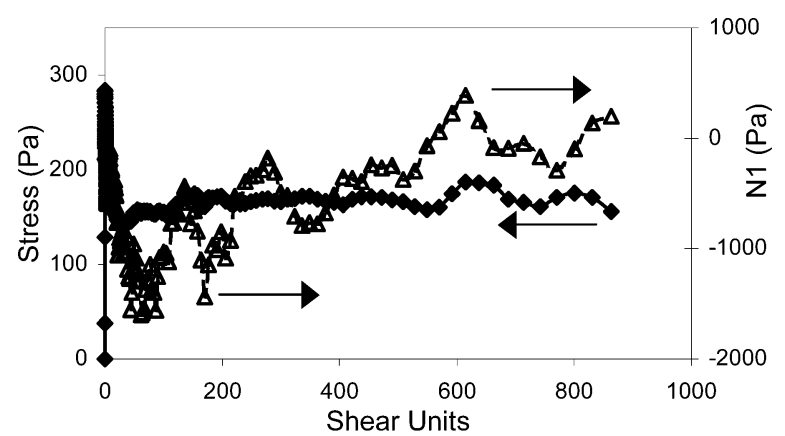

Figure 8. Response of $7.6 \mathrm{vol} \%$ (6 wt \%) SWNT in $102 \% \mathrm{H}_{2-}$ $\mathrm{SO}_{4}$ to start-up of shear flow in $25 \mathrm{~mm}$ parallel plates at a shear rate of $5 \mathrm{~s}^{-1}$. Solid diamonds represent shear stress, and open triangles represent $\mathrm{N} 1$.

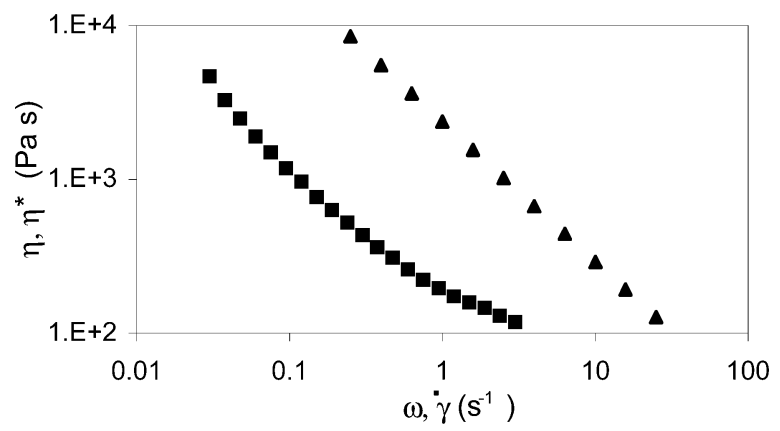

Figure 9. Cox-Merz rule is not obeyed. Example shown is $7.6 \mathrm{vol} \%$ (6 wt \%) SWNT in $102 \% \mathrm{H}_{2} \mathrm{SO}_{4}$. Triangles: dynamic viscosity, $\eta^{*}$, vs frequency, $\omega$. Squares: steady shear viscosity, $\eta$, vs shear rate, $\dot{\gamma}$.

equal when $\omega=\dot{\gamma}$. Lyotropic polymeric liquid crystals do not obey the Cox-Merz rule.

Aligned Solid Materials from Nematic Liquid Crystalline Phase. In addition to optical and rheological signatures in the liquid state, the nematic structure of SWNT/superacid dispersions is evidenced by scanning electron microscopy of materials quenched from the high-concentration dispersions. Scanning electron microscopy of ether-quenched SWNT/superacid dispersions shows that the SWNTs are free to rearrange into a variety of aligned species (Figure 10). Buckypapers prepared from SWNT/superacid solutions show the typical mat of entangled super-ropes. Such superropes are approximately 300-1000 nm wide, about 2 orders of magnitude wider than the original thin ropes (10-20 nm) generated by the H iPco reaction; the superropes come from the fast coagulation of liquid crystalline spaghetti domains which were not aligned with respect to each other. The introduction of moisture into the system deprotonates the nanotubes, reduces the electrostatic stabilization, and causes the precipitation of tactoid structures similar to those formed by inorganic liquid crystals of vanadium pentoxide. ${ }^{56}$ These structures are nearly monodisperse in dimension for a given starting concentration of SWNTs and show a high degree of alignment along their axis of 14:1 as measured by Raman. ${ }^{15}$ These tactoid structures have been named "alewives" for their resemblance to a Great Lakes fish. We believe that they are crystal solvate phases analogous to those formed by solutions of rodlike polymers in acids upon exposure to moisture. ${ }^{57}$ In the absence of moisture, the same starting concentration can be used to make highly aligned fibers with 20:1 axial to perpendicular alignment even in the absence of drawing ${ }^{34}$ as well as well-ordered sheets. ${ }^{58}$ 


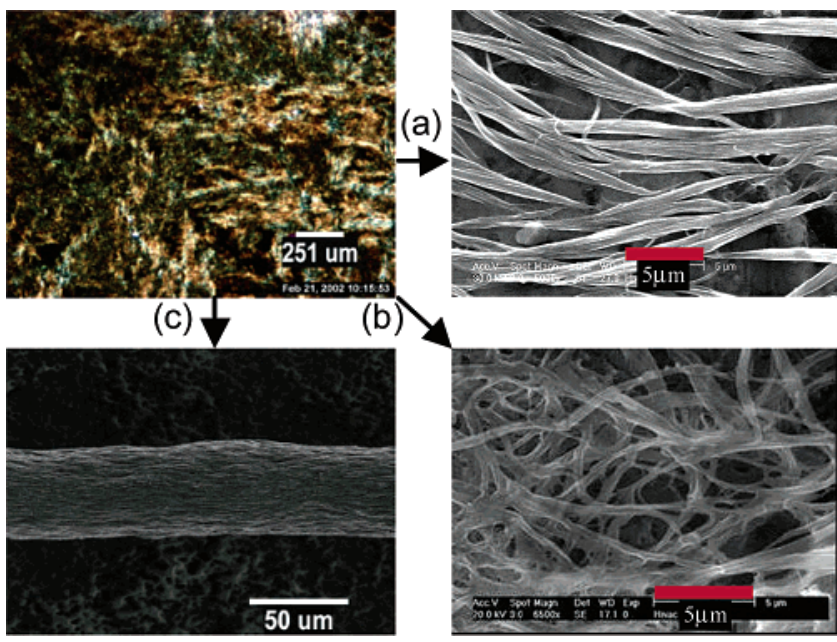

Figure 10. Rearrangement of SWNTs into aligned species. A liquid crystalline solution of SWNTs (e.g., $5.1 \mathrm{vol} \%$ or $4 \mathrm{wt}$ $\%$, top left) (a) can be phase-precipitated by introducing moisture into the dispersion, resulting in the formation of aligned species called alewives which have 14:1 alignment al ong their axis as measured by Raman; (b) can be quenched into ether and filtered, yielding a Bucky-paper of entangled ropes; (c) can be extruded under anhydrous conditions through a syringe needle, resulting in the formation of an al igned fiber with 20:1 alignment as measured by Raman (fiber extruded from 12.6 vol \% or 10 wt \% solution).

\section{Conclusions}

The protonation of single-walled carbon nanotubes in superacids allows them to be dispersed at high concentration, more than an order of magnitude higher than typical concentrations achieved in surfactants or organic solvents. The phase behavior of SWNTs in acid shows many parallels with that of Iyotropic nematogenic rodlike polymer solutions. The notable distinction is that in the biphasic region the anisotropic phase consists of extremely long self-assembled strands. At sufficiently high concentrations, approximately 4 vol $\%$ in $102 \% \mathrm{H}_{2}-$ $\mathrm{SO}_{4}$, a novel type of single phase nematic liquid crystal is formed. The nematic structure of SWNT/acid solutions is evidenced by the ability to form al igned species, birefringence, and rheological signatures including a maximum in the viscosity vs concentration curve, changes in sign of the first normal stress difference, and long oscillatory transients. In addition, the viscosity vs shear rate curve is suggestive of the three-region behavior associated with many lyotropic nematic polymeric liquid crystals. Exploiting successfully this phase behavior will be key to designing and optimizing a process for forming macroscopic objects comprised solely of highly aligned SWNTs.

Acknowledgment. We gratefully acknowledge use ful discussions with George Hirasaki, Satish Kumar, Gerry Lavin, Erik Hobbie, Karen Winey, J ack Fischer, Yachin Cohen, Ishi Talmon, Boris Yakobson, and Michael Wong. This work was funded by the DURINT initiative of the Office of Naval Research under Contract N0001401-1-0789, by NASA, by the Robert A. Welch foundation, and by the National Science Foundation. Additional support was provided by the Nanoscale Science and Engineering I nitiative of the National Science F oundation under NSF Award EEC-0118007.

\section{Appendix A}

The persistence length of SWNTs can be estimated by calculating the bending stiffness of a hollow cylinder 59

$$
\kappa=\frac{\pi C d^{3}}{8}
$$

and by using the relationship between persistence length and bending rigidity $\mathrm{L}_{\mathrm{p}} \equiv \kappa /\left(\mathrm{k}_{\mathrm{B}} \mathrm{T}\right)$. Taking $\mathrm{C}=$ $345 \mathrm{~N} / \mathrm{m}$ as characteristic in-plane rigidity of SWNTs ${ }^{60}$ and $\mathrm{d}=0.93 \mathrm{~nm}$ as characteristic HiPco diameter ${ }^{61}$ yields $L_{p} \approx 27 \mu \mathrm{m}$ at room temperature. In contrast, the persistence length of the stiffest rodlike polymers (e.g., PBO, PBZT, PBA, PBLG) is usually reported to range between 50 and $200 \mathrm{~nm}, 29,62$ approximately 2 orders of magnitude lower than the estimated persistence length of individual SWNTs. Calculations of SWNT persistence length based on the Young's modulus of ropes ${ }^{1}$ yield a value that is approximately 1 order of magnitude lower (a few micrometers); thus, the persistence length of SWNTs is 1-2 orders of magnitude higher than that of rodlike polymers.

\section{Appendix B}

If rodlike macromolecules are model ed as inextensible Brownian threads with finite bending stiffness, three dimensionless groups can be defined that characterize their behavior in a shear flow: the ratio of contour to persistence length (see Appendix A), the Weissenberg number, i.e., the product of the shear rate and rotational relaxation time $\mathrm{Wi} \equiv \dot{\gamma} \tau \equiv \delta_{\gamma} \mathrm{L}^{3} / 72 \mathrm{k}_{\mathrm{B}} \mathrm{T}$ (see eq 2 ), and the elasticity number El, i.e., ratio of viscous forces induced by the flow and elastic forces due to the bending stiffness, $\mathrm{EI} \equiv \dot{\gamma} \tau_{\mathrm{b}} \equiv \varsigma_{\gamma} \mathrm{L}^{4} / \alpha^{4} \kappa=\varsigma \dot{\gamma} \mathrm{L}^{4} / \alpha^{4} \mathrm{k}_{\mathrm{B}} \mathrm{TL} \mathrm{L}_{\mathrm{p}}$, where $\alpha$ $\approx 4.73$ is the eigenvalue of the most dangerous buckling eigenmode ${ }^{63,64}$ (wavelength $L$ ) -note that, within a numerical constant, the elasticity number is just the product of the Weissenberg number and the ratio of length to persistence length. Non-Brownian fibers in a shear flow can buckle when the elasticity number exceeds 0.44 (see ref 65 for experimental studies, ref 66 for the analytical criterion, and ref 67 for a numerical approximation). In Brownian fibers, buckling steepens the slope of the viscosity-shear rate curve; 68 buckling is controlled by a combination of the elasticity number and the ratio of length and persistence length because the ratio of length and persistence length controls the amplitude of the shape fluctuations, i.e., the initial amplitude of the buckling instability. ${ }^{68}$ For dilute SWNTs with $\mathrm{L} \approx 1.5 \mu \mathrm{m}$ and $\mathrm{L}_{\mathrm{p}} \approx 27 \mu \mathrm{m}$ we estimate that buckling should occur at Wi $\approx 1700$, i.e., $\dot{\gamma} \approx 2400$ $\mathrm{s}^{-1}$. Shorter SWNTs would buckle at higher shear rate.

\section{References and Notes}

(1) Baughman, R. H.; Zakhidov, A. A.; de Heer, W. A. Science 2002, 297, 787-792.

(2) Tans, S. J .; Devoret, M. H.; Dai, H.J .; Thess, A.; Smalley, R. E.; Geerligs, L. J .; Dekker, C. Nature (London) 1997, 386, 474-477.

(3) Hone, J .; Whitney, M.; Piskoti, C.; Zettl, A. Phys. Rev. B 1999, 59, R2514-R2516.

(4) Walters, D. A.; Ericson, L. M.; Casavant, M. J .; Liu, J Colbert, D. T.; Smith, K. A.; Smalley, R. E. Appl. Phys. Lett. 1999, 74, 3803-3805.

(5) Krishnan, A.; Dujardin, E.; Ebbesen, T. W.; Yianilos, P. N.; Treacy, M. M. J . Phys. Rev. B 1998, 58, 14013-14019.

(6) Buongiorno-Nardelli, M.; Yakobson, B. I.; Bernholc, J . Phys Rev. Lett. 1998, 81, 4656-4659. 
(7) Nikolaev, P.; Bronikowski, M. J .; Bradley, R. K.; Rohmund F.; Colbert, D. T.; Smith, K. A.; Smalley, R. E. Chem. Phys. Lett. 1999, 313, 91-97.

(8) Bronikowski, M. J .; Willis, P. A.; Colbert, D. T.; Smith, K. A.; Smalley, R. E. J . Vac. Sci. Technol. A: Vac. Surf. Films 2001, 19, 1800-1805.

(9) Zhu, H. W.; Xu, C. L.; Wu, D. H.; Wei, B. Q.; Vajtai, R.; Ajayan, P. M. Science 2002, 296, 884-886.

(10) Islam, M. F.; Rojas, E.; Bergey, D. M.; J ohnson, A. T.; Yodh, A. G. Nano Lett. 2002, 3, 269-273.

(11) Vigolo, B.; Poulin, P.; Lucas, M.; Launois, P.; Bernier, P. Appl. Phys. Lett. 2002, 81, 1210-1212.

(12) Vigolo, B.; Penicaud, A.; Coulon, C.; Sauder, C.; Pailler, R. J ournet, C.; Bernier, P.; Poulin, P. Science 2000, 290, 13311334.

(13) Dalton, A. B.; Collins, S.; Munoz, E.; Razal, J . M.; Ebron, V. H.; Ferraris, J. P.; Coleman, J . N.; Kim, B. G.; Baughman, R. H. Nature (London) 2003, 423, 703-703.

(14) Berry, G.; Cotts, P. M.; Chu, S. G. Br. Polym. J . 1981, 13, 47-54.

(15) Ramesh, S.; Ericson, L. M.; Davis, V. A.; Saini, R. K.; Kittrell C.; Pasquali, M.; Billups, W. E.; Adams, W. W.; Hauge, R. H.; Smalley, R. E. Submitted to J. Phys. Chem. B.

(16) Chiang, I. W.; Brinson, B. E.; Huang, A. Y.; Willis, P. A.; Bronikowski, M.J .; Margrave, J . L.; Smalley, R. E.; Hauge, R. H. J . Phys. Chem. B 2001, 105, 8297-8301.

(17) Kwolek, S. L.; Morgan, P. W.; Schaefgen, J . R.; Gulrich, L. W. Macromol ecules 1977, 10, 1390-1396.

(18) Bair, T. I.; Morgan, P. W.; Killian, F. L. Macromol ecules 1977 10, 1396-1400.

(19) Panar, M.; Beste, L. F. Macromolecules 1977, 10, 1401-1406.

(20) Onsager, L. Ann. N.Y. Acad. Sci. 1949, 51, 627-659.

(21) Flory, P. J. Proc. R. Soc. London, Ser. A 1956, 234, 73-89.

(22) Doi, M.; Edwards, S. F. The Theory of Polymer Dynamics; Oxford University Press: Oxford, 1986

(23) Kirkwood, J . G.; Auer, P. L. J . Chem. Phys. 1951, 19, 281283.

(24) Talmon, Y. Personal communication.

(25) Kirkwood, J . G.; Plock, R. J . J . Chem. Phys. 1956, 24, 665669.

(26) Batchelor. J Fluid Mech. 1970, 41, 545-557.

(27) Stepanek, I.; Parra-Vasquez, A. N. G.; Davis, V. A.; Pasquali, M. Manuscript in preparation. A length of $510 \mathrm{~nm}$ was extracted from an intrinsic viscosity of 10800 measured in surfactant-stabilized dispersions.

(28) Larson, R. G. TheStructureand Rheol ogy of Complex Fluids; Oxford University Press: New York, 1999.

(29) Mark, J. E. Polymer Data Handbook; Oxford University Press: New York, 1999.

(30) Berry, G. C.; Wong, C. P.; Venkatamen, S.; Chu, S. G. Technical Report, AFML-TR-79-4115, Air Force Materials Laboratory, Dayton, $\mathrm{OH}, 1979$.

(31) Roitman, D. B.; Wessling, R. A.; McAlister, J . Macromolecules 1993, 26, 5174-5184.

(32) Takada, Y.; Sato, T.; Teramoto, A. Macromol ecules 1991, 24, 6215-6219.

(33) Khokhlov, A. R. In Liquid Crystallinity in Polymers; Ciferri, A., Ed.; VCH Publishers: New York, 1991; pp 97-129.

(34) Ericson, L. M.; Davis, V. A.; Fan, H.; Wang, Y.; Vavro, J .; Zhou, W.; Guthy, C.; Sulpizio, J .; Ramesh, S.; Kittrell, C.; Fischer, J. E.; Pasquali, M.; Hauge, R. H.; Smalley, R. E. Manuscript in preparation.

(35) Kiss, G. D. In Rheology and Rheo-Optics of Concentrated Solutions of Helical Polypeptides. Ph.D. Thesis, University of Massachusetts, 1979; p 308.

(36) Kiss, G.; Porter, R. S. J. Polym. Sci., Part B: Polym. Phys. 1980, 18, 361-388.

(37) Kiss, G.; Porter, R. S. J . Polym. Sci., Part C: Polym. Symp. 1978, 193-211.
(38) Kiss, G.; Porter, R. S. In Mechanical and Thermophysical Properties of Polymer Liquid Crystals; Brostow, W., Ed.; Chapman \& Hall: London, 1998; pp 342-406.

(39) Lin, Q.; J ho, J .; Yee, A. F. Polym. Eng. Sci. 1993, 33, 789798.

(40) Wissbrun, K. F. J . Rheol. 1981, 25, 619-662.

(41) Qin, Y.; Brydon, D. L.; Mather, R. R.; Wardman, R. H. Polymer 1993, 34, 3597-3604.

(42) Onogi, S.; Asada, T. In Proceedings of theEighth I nternational Congress on Rheology; Astarita, G., Marrucci, G., Nicolais, L., Eds.; Plenum Press: Naples, Italy, 1980; pp 126-136.

(43) Baek, S. G.; Magda, J . J.; Larson, R. G.; Hudson, S. D. J . Rheol. 1994, 38, 1473-1503.

(44) Vavro, J .; Llaguno, M. C.; Fischer, J . E.; Ramesh, S.; Saini, R. K.; Ericson, L. M.; Davis, V. A.; Smalley, R. E. Phys. Rev. Lett. 2003, 90, Art. No. 065503.

(45) Laun, H. M. J . Non-Newtonian Fluid Mech. 1994, 54, 87108.

(46) Zarraga, I. E.; Hill, D. A.; Leighton, D. T. J . Rheol . 2000, 44, $185-220$.

(47) Aral, B. K.; Kalyon, D. M. J . Rheol. 1997, 41, 599-620.

(48) Montesi, A.; Pena, A. A.; Pasquali, M. Submitted to Phys. Rev. Lett.

(49) Lin-Gibson, S.; Pathak, J. A.; Grulke, E. A.; Wang, H.; Hobbie, E. K. Accepted in Phys. Rev. Lett.

(50) Grizzuti, N.; Cavella, S.; Cicarelli, P. J . Rheol. 1990, 34 $1293-1310$.

(51) Chaffey, C. E.; Porter, R. S. J . Rheol. 1985, 29, 281-305.

(52) Marrucci, G.; Maffetone, P. L. Macromolecules 1989, 22, 4076-4082.

(53) Larson, R. G.; Doi, M. J . Rheol. 1991, 35, 539-563.

(54) Larson, R. G. Macromol ecules 1990, 23, 3983-3992.

(55) Marrucci, G. In Liquid Crystallinity in Polymers; Ciferri, A., Ed.; VCH Publishers: New York, 1991; pp 395-421.

(56) Sonin, A. S. J . Mater. Chem. 1998, 8, 2557-2574.

(57) Cohen, Y.; Adams, W. W. Polymer 1996, 37, 2767-2774.

(58) Sreekumar, T. V.; Liu, T.; Kumar, S.; Ericson, L. M.; Hauge, R. H.; Smalley, R. E. Chem. Mater. 2003, 15, 175-178.

(59) Yakobson, B. I. In Handbook of Nanoscience, Engineering and Technology; Goddard III, W. A., Brenner, D. W., Lyshevsk, S. E., I afrate, G. J ., Eds.; CRC Press: New York, 2003.

(60) Kudin, K. N.; Scuseria, G. E.; Yakobson, B. I. Phys. Rev. B 2001, 6423, Art. No. 235406.

(61) Bachilo, S. M.; Strano, M. S.; Kittrell, C.; Hauge, R. H.; Smalley, R. E.; Weisman, R. B. Science 2002, 298, 23612366.

(62) Donald, A. M.; Windle, A. H. Liquid Crystalline Polymers; Cambridge University Press: Cambridge, 1992.

(63) Wiggins, C. H.; Riveline, D.; Ott, A.; Goldstein, R. E. Biophys. J. 1998, 74, 1043-1060.

(64) Shankar, V.; Pasquali, M.; Morse, D. C. J . Rheol. 2002, 46, $1111-1154$

(65) Forgacs, O. L.; Mason, S. G. J . Colloid Interface Sci. 1959 14, 457-472.

(66) Wiggins, C. H.; Montesi, A.; Pasquali, M. Manuscript in preparation

(67) Becker, L. E.; Shelley, M. J . Phys. Rev. Lett. 2001, 87.

(68) Montesi, A.; Pasquali, M.; Wiggins, C. H. Manuscript in preparation.

(69) Negative N1 has been observed occasionally in highly concentrated suspensions, ${ }^{45-47}$ once recently in emulsions, 48 occasionally in electrorheological suspensions under electric fields, 28 and also, very recently, in flocculated dispersions of multiwalled carbon nanotubes in toluene thickened with lowmolecular-weight polyisobutylene. ${ }^{49}$

MA0352328 